\section{CASE OF BRONZED SKIN, ASSOCIATED WITH DISEASE OF THE SUPRARENAL CAPSULES.}

By Join Kent Spender, Esq., Surgeon to the Eastern Dispensary, Bath.

[ical beforc the Bath and Bristol Branch, February 19th, 1857.]

Os the evening of the $3 \mathrm{rd}$ of October, 1856, I was called to visit a female, aged 21 , unmarried. I found her in bed, apparently very ill; and inquiry of her relatives elicited the following history of her complaint.

For nearly a year she had experienced an increasing sense of debility, which began without any obvious cause. There was nothing in her occupation which accounted for it; and her habits of life were regular and industrious. Menstruation had always been normal, and there was no record of leucorrhœa or of diarrhira. Progressive emaciation had accompanied the weakness, and a corresponding loss of animal heat; so that, although originally of good development, she had become exceedingly thin, and had felt very cold even during the excessive heat of last summer. There had been great lassitude, and hardly any appetite for food. Within the last three months, both herself and her friends had noticed a muddiness of skin on the face and hands; and, on the dorsal aspect of the latter, this muddiness had increased to such an extent that it might have been jmagined that she had exchanged limbs with a negress. The tint of the face was of a much less decided character. There was no history of any pulmonary or cardiac disease; and the surviving members of her family were in good health.

The first interview I had with my patient was by candlelight, and her appearance indicated nothing beyond aggravated spanæmia. It is not surprising that I should have overlooked, under such circumstances, the peculiar colour of the face; and the hands I did not particularly look at. The only thing which struck me was the extent and the deep hue of the areola round the eyes. I made a careful physical examination of the thoracic organs, but failed to discover anything abnormal beyond the usual anæmic murmurs. An examination of the abdomen led to only a negative result. No pain was complained of, but much nausea whenever she raised herself in bed; and, in the same posture, she described herself as experiencing a weight in the right hypochondriac region. The bowels were costive; but this fact was explicable by the small amount of nourishment taken. The urine was natural; the pulse was 80 ; the respirations 19 .

My patient had previously been under medical treatment, and, as far as I could learn, of a judicious kind. I was puzzled why she should have derived no permanent benefit from it, but I really could not see anything to interfere with a diagnosis of simple aggravated anæmia; and I offered rather an unguarded prognosis that she would probably be quite well in two or three weeks time. I prescribed a mixture of tincture of sesquichloride of iron with tincture of hyoscyamus, a dose after each meal; and directed that food should be given in small quanti. ties at frequent intervals.

October 4 th. I visited my patient by daylight, and I could not help noticing the dark earthy tint of her face and hands. No other portion of the surface was affected in the same way: the skin every where else was perfectly clear, and free from abnormal stain of any kind. However, the real nature of the case did not then occur to me. The medicine had caused a relaxation of the bowels, and therefore the citrate of iron was substituted for the sesquichloride; and an opiate pill ordered to be given at bedtime.

A decided improvement became manifest during the next few days, so that she was able to leave her bed and come down stairs, still preserving the recumbent posture on a sofa. On the joth of October, the feeling of weight in the right hypochondriac region increased to absolute pain. I made another physical examination of the abdomen, and the result was, that deep percussion elicited a dull note over the seat of pain, and palpation imparted the sense of a soft solid, deep in the abdo. minal cavity. Although the strength had increased, the emaciation progressively continued; ard, with a view to remedy this, I recommended the administration of the cod-liver oil. To this, however, great objection was offered, the bare thought of taking an oily substance appearing to create nausea.

October 13th. A retrograde step had already commenced. The citrate of iron mixture could no longer be retained; the bowels had become confined; the indescribable sense of power. lessness had completely returned; and my patient went back to her bed, from which she never rose again.

During the preceding week, I had been perusing Dr. Addison's celebrated monograph on Diseases of the Suprarenal Capsules, as well as a review of that work in the then current number of the British and Foreign Medico-Chirurgical Review. I now began to suspect the real nature of the malady; and $I$ at once communicated the possibilities and probabilities of the case to the patient's friends. They were astonished at the altered tone of my prognosis, and did not at all appreciate the reasons I gave for it. It was agreed, however, that further ad. vice should be obtained.

October 15th. Mr. Norman met me in consultation. It was clear that we had to deal with the results of a special dyscrasia, those results being manifested in a serious lesion of the nutritive processes. The data on which we had to form an opinion were principally subjective: these were the extreme lassitude and debility, the constant nausen and anorexia, the pain and the weight in the right hypochondrium, the depression of spirits, and the loss of memory. The great objective phenomena were two in number-the emaciation, and the bronzed colour of the face and hands. Our diagnosis appeared to be limited to the alternative of scrofulous degeneration of the intestinal glands, or disease of the suprarenal capsules, attended by its well known cutaneous discolorment. If the case had come under our notice some years ago, probably the former of these contingencies would have been the only one ta present itself; but, for obvious reasons, our opinion now decidedly inclined to the latter.

Mr. Norman strongly urged the administration of cod-liver oil, to which the patient reluctantly assented. Mr. Norman also suggested that a couple of grains of blue pill should be taken once or twice a week, not only with the view of maintaining a regular action of the bowels, but also to assist the func tions of the liver ; for the possibility of biliary toxæmia had oc curred to our minds as tending to confuse the other elements of diagnosis; although such an hypothesis would not accoun for the emaciation, and was almost certainly negatived by the clear pearly hue of the conjunctiva.

Under the plan of treatment above laid down, and for some time faithfully carried out, the tendency to emaciation was certainly arrested, and the strength appeared to be somewhat im proved. A blister was subsequently applied to the right hypochondrium, in order to relieve the local pain.

Things remained very much in statu quo until the middle of November. Disgust of the cod-liver oil was now experienced and consequently its use was abandoned; and so complete was the repugnance to every kind of food, that beef-tea, in ver limited quantities, was the only article of diet which we could persuade her to accept. The sense of hunger was altogether suspended, and the withdrawal of the demand for nourishmen was apparently the proximate cause of that wasting process which now continued uninterruptedly until death. And yet the secretions generally were properly carried on; there was 11 cough, and no symptom referrible to the pulmonary organs the sounds of the heart were sharp and clear; and, with the exception of the faculty of memory, the intellectual powers were unimpaired.

For a short time, I gave her quinine, with a slight improvement of the appetite; but, at the beginning of December, she firmly refused to take medicine of any kind. A week afterwards, she would see nobody beyond her own family; and on Friday, December 19th, she quietly died away, as if she had only fallen asleep.

Post Mortem Examination, December 21st, fifty-fours after death. The body was greatly wasted; the breasts very much atrophied; there was very little adipose tissue anywhere; and the muscular structures were flabby and attenuated. The skin of the face was nearly as brown as a mulatto: the exact hue it would be difficult to describe, but it was duller and less intense than bronze, and resembled more than anything else the tint of the lighter portions of a photographic impression. The colour was very marked when contrasted with the integument of the thorax and abdomen, or with any linen article of clothing. The skin of the dorsal aspect of the hand was exceedingly. dark, the knuckles being particularly so: here the word "bronze" most fitly conveys the real tint, because it really possessed the shining properties of that alloy. The popular term "brown jaundice" is sometimes a very appropriate one in these cases.

The cranium was not examined. The viscera of the thorax were anatomically healthy, but very bloodless: the heart was exceedingly small, and contained several fibrinous coagula. The stomach was distended with gas: it contained a thin 
yellowish fluid, but its coats were in all respects free from disease. Nothing abnormal could be detected in the large and small intestines; the spleen and the liver were of the natural size. When the liver was being removed, I found that the right kidney was dragged out with it; the fact being that an adhesion had taken place between the contiguous peritoneal layers of the posterior lobe of the liver and of the suprarenal capsule. This circumstance may adequately account for the constant pain in the right hypochondriac region during life; and the sense of weight also complained of was evidently caused by the entire mass unduly stretching the ligaments by which the liver is attached to the diaphragm. A slow process of adhesive inflammatory action had probably been going on for some time: the connection between the two organs was of the most intimate kind, and the knife was required to separate them. The right suprarenal capsule was very large: the left scarcely exceeded the normal size, and was unadherent to adjacent viscera. Purulent matter exuded from both glands when a section was made through them: and a number of little miliary bodies was visible to the naked eve. Ny friend, Dr. Martyn, of Bristol, has been kind enough to make a careful examination of the diseased organs. His report is as follows:- "Left suprarenal capsule. Preservation good, colour reddish-yellow, surface uneven: weight 75 grains. Tears readily, showing rough yellowish-white surfaces, covered with creamy fluid. Consists of a thickened tunic, within which are fibrous septa, forming meshes in which cheesy masses lie loosely surrounded by creamy fluid. Microscopic appear ance. (a) Irregularly roundish nuclear corpuscles, containing fatty granules (often to half the entire cell-contents). (b) Parent-cells containing (a). (c) Proteine granules. (d) Large free granules of fat. (c) Fusiform cells, also containing fat. These are derived from the septa. The right suprarenal body exhibited a similar condition Kidney pale. From the anpearances above-mentioned, and from the action of glycerine, acetic acid, and ether, I conclude that the specimen is one of yellow tubercle in an advanced stage of degenerative softening."

The specimen has been deposited in the museum attached to the Bristol Medical School.

ReMarks. On looking through a valuable series of cases in the Medical Times and Gazette, illustrative of disease of the suprarenal capsules, I find that there are three symptoms more commonly present than any others. These are debility, bronzed skin, and emaciation. I have enumerated them in the order of frequency. The debility appears to exist always. It indicates the development of a special dyscrasia, characterised by a grave error in the blood forming process, and con sequent prostration of the vital powers. The dyscrasia is one which probably has some affinity with idiopathic anæmia, with chlorosis, or with that constitutional state which precedes the local deposit of tubercle. It is impossible in all cases to define its cause: but it is certain that some mental derangement or shock has had a great deal to do with many of the cases of this disease which have been published. Solidists and humoralists may here find a capital debating ground: for the inquiry is naturally suggested, is the disease of the blood the cause of the neurosis, or the neurosis the cause of the blood-disease?

Next let me advert to the bronzed skin. I find two appa rently authentic cases on record in which bronzing of the skin was unaccompanied by any noticeable changes in the suprarenal capsules. But these are only two cases out of a considerable number in which the disease of the glands has been successfully diagnosed during life from the cutaneous discoloration. Partial disease of the capsules has been detected in several instances without any corresponding bronzing; but I believe that not a single case has been published in which total destruction of the capsules has existed, without manifest discoloration of the integuments also. Hence, as the British and Foreign Reviewer remarks, the cutaneous bronzing may be dependent upon the capsular affection, but the converse of this proposition cannot for a moment be entertained. But while I agree with the Reviewer in thinking that the condition of the integument is almost absolutely diagnostic of disease of the capsules, I think he too hastily concludes that the latter is the cause of the former. We must know a great deal more than we do now about the physiology of the ductless glands, before we venture to pronounce so decisively on the phenomena of their pathology.

Emaciation is a symptom not so frequently observed, and in many cases has been entirely absent. It is easily explained, whenever it exists, by the depraved condition of the blood, and by the altered nerve-tone resulting therefrom. Nutrition must be gravely interfered with by such primary elements of disease.

The case which I have related affords a marked illustration of the three great symptoms. The debility and emaciation existed in an extreme degree; but the bronzing was strictly limited to those portions of the skin habitually exposed to the light, and therefore looked like an aggravated form of sunburntness.

Dr. Addison's opinion as to the hopelessness of the prognosis appears to be confirmed by every fresh case which is published. 'The treatment is unsatisfactory in every sense of the word: nerve-tonics and blood-tonics are equally useless; and perhans we shall hare to await the discovery of a new specific before being able to announce our ability to control the progress of this singular disease.

An account of the structural anatomy of the suprarenal capsules, by Kölliker, is to be found in the Medical Times and Gazette, vol. ii, for 1855. M. Brown-Séquard's investigations in their physiology show that these bodies have a very important part to play in the animal economy - a part which, if omitted, must lead to fatal results. (Arch. Générales de Med. Oct. and Nov. 1850, quoted in Ranking's Abstract, Jan. 1857.) The substance of M. B.-Séquard's observations is, that $(a)$ the capsules are very sensitive. (b) As age adrances, the capsules are found to gain considerably both in weight and in volume; and hence it appears that these organs are not exclusively related to embryonic life. (c) Extirpation of both capsules destroys life with as much certainty, and with greater rapidity than extirpation of the kidneys. The extirpation of a single organ was invariably fatal. After removal of both capsules, the following phenomena were noticed: feebleness, gradually passing to extreme prostration; a respiration first quickened then retarded, and lastly irregular and spasmodic; a quick and weakened pulse; gradual diminution of animal heat; and lastly, vertigo, convulsions in various forms, and occasionally coma. It would thus appear that the suprarenal capsules are essential to life, and that their removal or disorganisation may lead partly to some injurious alteration in the blood, and partly to some injurious operation on the nervous system.

\section{CASE OF WOUND OF KIDNEY : RECOVERY.}

By J. Johnston, M.B.Lond.

[Read before the Birmingham and Midland Counties Branch, February 12th, 1857.]

ON December 24th, 1855, at 10 P.Mr., I was hastily summoned to attend William Hyde, aged 32 , who had been stabbed in quarrel. When I arrived at the public house at which he lay, I found him in a state of collapse from the great loss of blood; the pulse hardly perceptible; vomiting was continuous, with hiccup. He complained of severe but spasmodic pain in the groin, where, he said, he had been stabbed with a carving knife. I therefore examined the groin: there was no wound, but a spasmodic retraction of the testicle. Having turned him on to his side, I found an incised wound, one inch and a half in length, on the right side of the spinal column, and about two and a half inches above the posterior crest of the ilium. Con. siderable hæmorrhage was still taking place, and he said that the knife had broken in his back. Having sent the policeman to find the broken knife, during his absence I assiduously applied cold water to the lumbar region, and gave the patient some brandy and cold water, with forty drops of tincture of opium. The policeman not being able to recover the knife, I thought it advisable to pass my finger into the wound, to probe for any broken piece. My finger passed upwards and inwards, touching the transverse process of the third lumbar vertebra, and about an inch above this entered the peritoneal cavity. The knife was afterwards found, unbroken, but bent about three-fourths of an inch from the point: it was eight and a half inches in length, and had been driven in up to the haft. I continued the cold application. A magistrate having arrived, the patient's deposition was taken, as I did not think that he could rally. Warmth was applied to the feet. Gallic acid in five-grain doses, with a fourth of a grain of opium, were given every twenty minutes, for three doses, and afterwards repeated every hour, with occasional doses of cold brandy and water. He chiefly complained of retraction and pain in the testicle; this was relieved by an embrocation of olive oil and tincture of opium in equal parts. About half an hour after my arrival, he expressed a desire to pass his urine, and then passed by the 